\title{
SEMISTABLE REDUCTION FOR OVERCONVERGENT $F$-ISOCRYSTALS ON A CURVE
}

\author{
KIRAN S. KEDLAYA
}

\begin{abstract}
Let $X$ be a smooth affine curve over a field $k$ of characteristic $p>0$ and $\mathcal{E}$ an overconvergent $F^{a}$-isocrystal on $X$ for some positive integer $a$. We prove that after replacing $k$ by some finite purely inseparable extension, there exists a finite separable morphism $X^{\prime} \rightarrow X$, the pullback of $\mathcal{E}$ along which extends to a $\log -F^{a}$-isocrystal on a smooth compactification of $X^{\prime}$. This resolves a weak form of the global version of a conjecture of Crew; the proof uses the local version of the conjecture, established (separately) by André, Mebkhout and the author.
\end{abstract}

\section{Introduction}

The purpose of this paper is to prove the following semistable reduction theorem for overconvergent $F$-isocrystals on a curve, answering a conjecture of de Jong [dJ, Section 5], a reformulation of a conjecture of Crew (of which more about below).

Theorem 1.1 (Semistable reduction). Let $X$ be a smooth, geometrically connected curve over a field $k$ of characteristic $p>0$, let $K$ be a complete discrete valuation field with residue field $k$, admitting a lift of the p-power Frobenius on $k$, and let $\mathcal{E}$ be an overconvergent $F^{a}$-isocrystal on $X$ with respect to $K$ for some $a \in \mathbb{N}$. Then after replacing $k$ by a suitable finite purely inseparable extension (depending on $\mathcal{E}$ ), there exist a finite generically étale morphism $f: X_{1} \rightarrow X$, a smooth compactification $j: X_{1} \hookrightarrow \bar{X}_{1}$ of $X_{1}$, and a log-F ${ }^{a}$-isocrystal $\mathcal{F}$ on $\left(\overline{X_{1}}, \overline{X_{1}} \backslash X_{1}\right) / K$ such that $j^{*} \mathcal{F} \cong f^{*} \mathcal{E}$.

Our basic approach is to use the quasi-unipotence theorem ( $p$-adic local monodromy theorem) for $F^{a}$-isocrystals, plus a matrix factorization argument from [Ke1], to "fill in" $\mathcal{E}$ at each of the points of $\overline{X_{1}} \backslash X_{1}$. The quasi-unipotence theorem, conjectured by Crew [Cr, Section 10], follows from the work of any of André $[\mathrm{A}]$, Mebkhout [M], or the author [Ke2]; see Proposition 3.1 for the formulation we need here.

Theorem 1.1 "almost" yields a more precise statement proposed by Crew $[\mathrm{Cr}]$, by implying that there exists a finite morphism $f: X_{1} \rightarrow X$ such that $f^{*} \mathcal{E}$ is unipotent at each point of $\overline{X_{1}} \backslash X_{1}$. (In fact, we will prove Theorem 1.1 by proving this first.) The caveat is that Crew actually wanted $f$ to be étale.

Received April 9, 2002. 
We have only been able to achieve this in the unit-root case, and do not know whether it should be possible in general. We discuss these matters in Section 6 .

We hope to extend Theorem 1.1 to higher dimensional varieties in subsequent work. In this case, we must allow the morphism $f: X_{1} \rightarrow X$ to be generically étale, but not necessarily finite, because the normalization of $X$ in a finite extension of its function field need not be smooth. For this and other reasons, the argument in higher dimensions will be technically more involved.

The precise higher-dimensional analogue of Theorem 1.1 has been formulated conjecturally by Shiho [S, Conjecture 3.1.8]; by Shiho's work, proving this statement would provide good comparison results between rigid and crystalline cohomology. For example, it would yield an alternate proof of finite dimensionality of rigid cohomology of a curve with coefficients in an overconvergent $F$-isocrystal, via comparison with crystalline cohomology; Theorem 1.1 does this for curves. (Crew's proof in $[\mathrm{Cr}]$ uses $p$-adic functional analytic techniques; the general finiteness proof in [Ke3] uses a devissage to the curve case.)

\section{Definitions and notations}

We set up notation following [Ke3]; this makes it a bit more convenient to work with global objects than does the notation of [Ke2]. We retain the convention of [Ke2] that all matrices are $n \times n$ matrices and $I$ denotes the identity matrix.

Let $k$ be a field of characteristic $p>0$, and let $C(k)$ be a Cohen ring for $k$, that is, a complete discrete valuation ring with residue field $k$ and maximal ideal generated by $p$. (See [Bo] for proof of existence and basic properties of Cohen rings.) Let $K$ be a finite totally ramified extension of Frac $C(k)$, let $\mathcal{O}$ be the integral closure of $C(k)$ in $K$, and let $v_{p}$ denote the $p$-adic valuation on $K$. Assume that there exists a ring endomorphism $\sigma_{0}$ on $\mathcal{O}$ lifting the $p$-power Frobenius on $k$. Let $q=p^{a}$ be a power of $p$, and put $\sigma=\sigma_{0}^{a}$.

The ring $\mathcal{R}_{r}$ consists of bidirectional power series $\sum_{i \in \mathbb{Z}} c_{i} u^{i}$, with $c_{i} \in K$, such that

$$
\lim _{i \rightarrow \pm \infty} s v_{p}\left(c_{i}\right)+i=\infty \quad(0<s \leq r)
$$

for each $s$, the function $w_{s}\left(\sum_{i} c_{i} u^{i}\right)=\min _{i}\left\{s v_{p}\left(c_{i}\right)+i\right\}$ is a nonarchimedean valuation on $\mathcal{R}_{r}$. The ring $\mathcal{R}$ (the "Robba ring") is the union of the $\mathcal{R}_{r}$ over all $r>0$. Its subring $\mathcal{R}^{\text {int }}$ consists of those series with $c_{i} \in \mathcal{O}$ for all $i$; this subring is a (noncomplete) discrete valuation ring, unramified over $\mathcal{O}$, with residue field the field $k((t))$ of formal Laurent series in $k$. (In [Ke2], the rings $\mathcal{R}$ and $\mathcal{R}^{\text {int }}$ are called $\Gamma_{\text {an,con }}$ and $\Gamma_{\text {con }}$, respectively.) By adding the superscript + or - to $\mathcal{R}$ or $\mathcal{R}^{\text {int }}$, we will mean the subring with only nonnegative or nonpositive powers of $u$, respectively.

For $L / k((t))$ finite separable, there is a natural discrete valuation ring $\mathcal{R}_{L}^{\text {int }}$, integral and unramified over $\mathcal{R}^{\text {int }}$, with residue field $L$. Namely, take any monic polynomial $P(x)$ over $\mathcal{R}^{\text {int }}$ whose reduction $\bar{P}$ satisfies $K \cong k((t))[x] /(\bar{P}(x))$, and put $\mathcal{R}_{L}^{\text {int }} \cong \mathcal{R}^{\text {int }}[x] /(P(x))$. 
The Monsky-Washnitzer algebra of rank $n$ is defined as

$$
W_{n}=\left\{\sum_{I} c_{I} x^{I}: c_{I} \in \mathcal{O}, \quad \liminf _{I} \frac{v_{p}\left(c_{I}\right)}{\sum I}>0\right\},
$$

where $I=\left(i_{1}, \ldots, i_{n}\right)$ represents an $n$-tuple of nonnegative integers, $x^{I}=$ $x_{1}^{i_{1}} \cdots x_{n}^{i_{n}}$ and $\sum I=i_{1}+\cdots+i_{n}$. An integral dagger algebra is any quotient $A^{\text {int }}$ of a Monsky-Washnitzer algebra which is flat over $\mathcal{O}$ and for which $\operatorname{Spec}\left(A^{\text {int }} \otimes_{\mathcal{O}} k\right)$ is smooth over $\operatorname{Spec}(k)$. (Given $A^{\text {int }} \otimes_{\mathcal{O}} k$, one can always find a corresponding $A^{\text {int }}$; see $[\mathrm{vdP}]$.) A dagger algebra $A$ is an algebra of the form $A^{\text {int }} \otimes_{\mathcal{O}} K$ for some integral dagger algebra $A^{\text {int }}$ (uniquely determined by $A$ and the $p$-adic valuation on $A$ ).

Given a dagger algebra $A$ with $A^{\text {int }} \cong W_{n} / \mathfrak{a}$ and $f \in A$ not a zero divisor, the localization $A^{\prime}$ of $A$ at $f$ is the dagger algebra with

$$
\left(A^{\prime}\right)^{\mathrm{int}} \cong W_{n+1} /\left(W_{n+1} \mathfrak{a}+\left(f x_{n+1}-1\right) W_{n+1}\right) ;
$$

this is a dagger algebra in which $f$ is invertible.

Given a dagger algebra $A$ with $A^{\text {int }} \cong W_{n} / \mathfrak{a}$ for some ideal $\mathfrak{a}$ of $W_{n}$, we define $\Omega_{A / K}^{1}$ as the free $A$-module generated by symbols $d x_{1}, \ldots, d x_{n}$, modulo relations of the form $d a=0$ for $a \in \mathfrak{a} \otimes_{\mathcal{O}} K$. By construction, $\Omega_{A / K}^{1}$ is equipped with a $K$-linear derivation $d: A \rightarrow \Omega_{A / K}^{1}$.

\section{Log- $F$-isocrystals and $\log -(\sigma, \nabla)$-modules}

Let $A$ be a dagger algebra and $\sigma_{0}: A^{\text {int }} \rightarrow A^{\text {int }}$ a lift of the $p$-power Frobenius map extending the given $\sigma_{0}$ on $\mathcal{O}$; again, set $\sigma=\sigma_{0}^{a}$. (Such a lift always exists: again, see $[\mathrm{vdP}]$.) Given $u \in A$ such that $u^{\sigma} / u^{q}$ is invertible in $A$, we define the logarithmic module of differentials $\Omega_{A / K}^{1}[d \log u]$ by adding to $\Omega_{A / K}^{1}$ a symbol $d \log u$ such that $u(d \log u)=d u$; then $d \sigma$ extends to $\Omega_{A / K}^{1}[d \log u]$ sending $d u / u$ to $q d u / u+d\left(u^{\sigma} / u^{q}\right) /\left(u^{\sigma} / u^{q}\right)$. We define a log- $(\sigma, \nabla)$-module over $A$ (with respect to $u$ ) as a finite locally free $A$-module $M$ equipped with a $\sigma$ linear map $F$ that induces an isomorphism $F: M \otimes_{A, \sigma} A \rightarrow M$, and with an $A$-linear connection $\nabla: M \rightarrow M \otimes_{A} \Omega_{A / K}^{1}[d \log u]$ which is integrable (i.e., which satisfies $\nabla_{1} \circ \nabla=0$ for $\nabla_{1}: M \otimes \Omega_{A / K}^{1}[d \log u] \rightarrow M \otimes \wedge_{A}^{2} \Omega_{A / K}^{1}[d \log u]$ induced by $\nabla$ ) and which makes the following diagram commute:

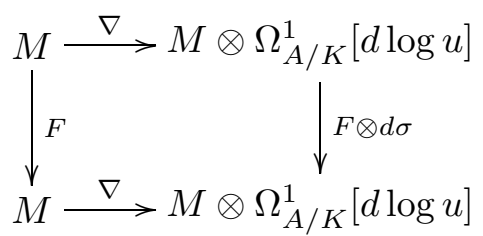

If $u=1$, we drop the "log" and simply refer to $M$ as a $(\sigma, \nabla)$-module. We analogously define $(\sigma, \nabla)$-modules over $\mathcal{R}$ for $\sigma: \mathcal{R} \rightarrow \mathcal{R}$ induced by the $a$-th 
composition power of a map $\sigma_{0}: \mathcal{R}^{\text {int }} \rightarrow \mathcal{R}^{\text {int }}$ of the form

$$
\sum_{i} c_{i} u^{i} \mapsto \sum_{i} c_{i}^{\sigma_{0}}\left(u^{\sigma_{0}}\right)^{i}
$$

lifting the $p$-th power map. In this case, we take $\Omega_{\mathcal{R} / K}^{1}$ to be the free $\mathcal{R}$-module generated by $d u$. (Note: since we are only considering curves, the integrability condition will be superfluous in our situations, as $\wedge^{2} \Omega^{1}$ will vanish.)

Log- $(\sigma, \nabla)$-modules are Zariski-local avatars of more global objects, namely overconvergent $\log -F^{a}$-isocrystals. Rather than take space for a full-blown definition of $\log -F^{a}$-isocrystals here, we summarize the key features of the definition below.

- If $X$ is smooth over $k$ and equipped with the fine log structure associated to some strict normal crossings divisor $Z$, there is a category of overconvergent $\log -F^{a}$-isocrystals on the pair $(X, Z)$. (We drop the "log" if $Z$ is empty.)

- An oveconvergent log- $F^{a}$-isocrystal on $(X, Z)$ can be specified by giving overconvergent $\log -F^{a}$-isocrystals on an affine cover of $X$ plus isomorphisms on the pairwise intersections satisfying the cocycle condition. (Loosely put, the category is a Zariski sheaf.)

- Given a dagger algebra $A$, a Frobenius lift $\sigma$, and an element $u \in A^{\text {int }}$ such that $u^{\sigma} / u^{q}$ is invertible in $A$, the category of $\log$ - $(\sigma, \nabla)$-modules with respect to $u$ is canonically equivalent to the category of overconvergent log$F^{a}$-isocrystals on $(X, Z)$, where $X=\operatorname{Spec}\left(A^{\text {int }} \otimes_{\mathcal{O}} k\right)$ and $Z \subseteq X$ is the zero locus of $u$. In particular, the former does not depend on $\sigma$; in fact, there is an explicit formula for transforming a Frobenius structure with respect to a given $\sigma$ into a Frobenius structure with respect to another (with respect to the same $\nabla$ ).

See [CI, Section 6] for an informal overview of $\log -F^{a}$-isocrystals; for a much more detailed study, see [S, Chapter 2]. (It might help to consider the situation without logarithmic structures first; see [Be] for an introduction there.)

The main input into this paper is the $p$-adic local monodromy theorem ("Crew's conjecture"), established separately by André [A], Mebkhout [M], and the author [Ke2]. We say an extension of $k((t))$ is nearly finite separable if it is finite separable over $k^{1 / p^{n}}((t))$ for some nonnegative integer $n$. With that definition, the local monodromy theorem (e.g., in the form of [Ke2, Theorem 6.12]) implies the following.

Proposition 3.1. Let $M$ be a $(\sigma, \nabla)$-module over $\mathcal{R}$. Then there exists a nearly finite separable extension $L / k((t))$ so that $M \otimes_{\mathcal{R}^{\text {int }}} \mathcal{R}_{L}^{\text {int }}$ admits a basis $\mathbf{v}_{1}, \ldots, \mathbf{v}_{n}$ such that $\nabla \mathbf{v}_{i} \in \operatorname{SatSpan}\left(\mathbf{v}_{1}, \ldots, \mathbf{v}_{i-1}\right) \otimes \Omega^{1}$ for $i=1, \ldots, n$.

In fact, if $u \in \mathcal{R}_{L}^{\text {int }}$ lifts a uniformizer of $L$ and $K^{\prime}$ is the integral closure of $K$ in $\mathcal{R}_{L}^{\text {int }}$ (so that $\mathcal{R}_{L}^{\text {int }}$ is isomorphic to the integral Robba ring with coefficient field $\left.K^{\prime}\right)$, then one can ensure that in fact $\nabla \mathbf{v}_{i} \in\left(K^{\prime} \mathbf{v}_{1}+\cdots+K^{\prime} \mathbf{v}_{i-1}\right) \otimes \frac{d u}{u}$; this implies that $F \mathbf{v}_{i} \in K^{\prime} \mathbf{v}_{1}+\cdots+K^{\prime} \mathbf{v}_{n}$ for all $i$. If such a basis already exists over $\mathcal{R}$, we say $M$ is unipotent over $\mathcal{R}$. 


\section{Matrix factorizations}

In this section, let $A$ be a dagger algebra such that $\operatorname{Spec}\left(A \otimes_{\mathcal{O}} k\right)$ is a smooth affine geometrically connected curve over $\operatorname{Spec}(k)$; in particular, $A$ is an integral domain. Suppose $t \in A \otimes_{\mathcal{O}} k$ generates a prime ideal with residue field $k$; choose a lift $u \in A^{\text {int }}$ of $t$. Then there is a natural embedding $\rho_{u}: A^{\text {int }} \hookrightarrow \mathcal{R}^{+ \text {,int }}$ sending $u$ to the series parameter; this extends to an embedding of any localization of $A$ into $\mathcal{R}$. We identify each localization of $A$ with its image under $\rho_{u}$.

Lemma 4.1. For $r>0$, let $U$ be an invertible matrix over $\mathcal{R}_{r}$. Then there exists an invertible matrix $V$ over some localization $A^{\prime}$ of $A$ such that $w_{r}(V U-I)>0$.

Proof. Let $A_{1}$ be the localization of $A$ at $u$; then $A_{1}$ is dense in $\mathcal{R}_{r}$ under $w_{r}$. Thus we can choose a matrix $V$ over $A_{1}$ such that $w_{r}\left(V-U^{-1}\right)>-w_{r}(U)$; then

$$
w_{r}(V U-I)=w_{r}\left(\left(V-U^{-1}\right) U\right) \geq w_{r}\left(V-U^{-1}\right)+w_{r}(U)>0 .
$$

Since $w_{r}(V U-I)>0$, we have $w_{r}(\operatorname{det}(V U)-1)>0$, so in particular $\operatorname{det}(V U) \neq$ 0 . Hence $\operatorname{det}(V) \neq 0$, so we can form the localization $A^{\prime}$ of $A_{1}$ at $\operatorname{det}(V)$. Over $A^{\prime}, V$ becomes an invertible matrix, as desired.

Proposition 4.2. Let $U$ be an invertible matrix over $\mathcal{R}$. Then there exist invertible matrices $V$ over some localization $A^{\prime}$ of $A$ and $W$ over $\mathcal{R}^{+}$such that $U=V W$.

Proof. Choose $r>0$ so that $U$ is invertible over $\mathcal{R}_{r}$. By Lemma 4.1, we can find an invertible matrix $X$ over some localization $A_{1}$ of $A$ such that $w_{r}(X U-I)>0$. By [Ke2, Proposition 6.5], we can write $X U$ as a product $Y Z$ with $Y$ invertible over $\mathcal{R}^{-}$and $Z$ invertible over $\mathcal{R}^{+}$. Let $A^{\prime}$ be the localization of $A_{1}$ at $u$; then $\mathcal{R}^{-} \subseteq A^{\prime}$, so $X^{-1} Y$ is invertible over $A^{\prime}$. Thus we may take $V=X^{-1} Y$ and $W=Z$.

\section{Semistable reduction}

In this section, we prove that an overconvergent $F^{a}$-isocrystal $\mathcal{E}$ on $X$ which is unipotent at each point of $\bar{X}-X$, for $\bar{X}$ a smooth compactification of $X$, has "semistable reduction." This will yield our proof of Theorem 1.1.

We begin with a result that translates unipotence of a $(\sigma, \nabla)$-module into semistable reduction. The argument is based on the proof of $[\mathrm{Ke} 1$, Theorem 5.0.1].

Theorem 5.1. Let $A$ be a dagger algebra equipped with a Frobenius lift $\sigma$. Suppose the image of $u \in A^{\mathrm{int}}$ in $A^{\mathrm{int}} \otimes_{\mathcal{O}} k$ generates a prime ideal and $u^{\sigma} / u^{q}$ is a unit in $A$. Let $A^{\prime}$ be the localization of $A$ at $u$, and let $M$ be a free $(\sigma, \nabla)$-module over $A^{\prime}$ which becomes unipotent over $\mathcal{R}$ (where $A^{\prime}$ is identified with a subring of $\mathcal{R}$ via $\left.\rho_{u}\right)$. Then $M$ is isomorphic to a $\log -(\sigma, \nabla)$-module, with respect to $u$, over some localization $A^{\prime \prime}$ of $A$ in which $u$ is not invertible. 
Proof. Let $\mathbf{e}_{1}, \ldots, \mathbf{e}_{n}$ be a basis of $M$, so that $F \mathbf{e}_{j}=\sum_{i} \Phi_{i j} \mathbf{e}_{i}$ and $\nabla \mathbf{e}_{j}=$ $\sum_{i} N_{i j} \mathbf{e}_{i} \otimes \frac{d u}{u}$. Define the differential operator $\theta(f)=u \frac{d f}{d u}$. By hypothesis, there exists a matrix $U$ over $\mathcal{R}$ such that $U^{-1} \Phi U^{\sigma}$ and $U^{-1} N U+U^{-1} \theta(U)$ have entries in $\mathcal{O}$. By Proposition 4.2, we can factor $U$ as $V W$, where $V$ is invertible over some localization $A_{1}$ of $A^{\prime}$ and $W$ is invertible over $\mathcal{R}^{+}$. Now put $\mathbf{v}_{j}=\sum_{j} V_{i j} \mathbf{e}_{i}$; then $F \mathbf{v}_{j}=\sum_{i} \tilde{\Phi}_{i j} \mathbf{v}_{i}$ and $\nabla \mathbf{v}_{j}=\sum_{i} \tilde{N}_{i j} \mathbf{v}_{i} \otimes \frac{d u}{u}$, where

$$
\begin{aligned}
& \tilde{\Phi}=V^{-1} \Phi V^{\sigma}=W\left(U^{-1} \Phi U^{\sigma}\right) W^{-\sigma} \\
& \tilde{N}=V^{-1} N V+V^{-1} \theta(V)=W\left(U^{-1} N U+U^{-1} \theta(U)\right) W^{-1}-\theta(W) W^{-1}
\end{aligned}
$$

have entries in $A^{\prime \prime}=A_{1} \cap \mathcal{R}^{+}$, which is a localization of $A$ (because it contains $A$ and is contained in the localization $A_{1}$ ) in which $u$ is not invertible (because $u$ is not invertible in $\mathcal{R}^{+}$).

We now proceed to the proof of our main theorem.

Proof of Theorem 1.1. Let $K(X)$ be the function field of $X$, and let $\bar{X}$ be a smooth compactification of $X$. Without loss of generality, enlarge $k$ so that the geometric points of $Z=\bar{X} \backslash X$ are $k$-rational. (Given the desired result over a finite extension of $k$, we deduce the result over $k$ by restriction of scalars.) For each geometric point $x$ of $Z$, choose a function $t_{x} \in K(X)$ with a simple zero at $x$, and choose an open affine neighborhood $U_{x}$ of $x$ in $\bar{X}$ such that $U_{x} \cap Z=\{x\}$ and $\operatorname{div}\left(t_{x}\right) \cap U_{x}=\{x\}$. Let $A_{x}$ be a dagger algebra with $U \cong \operatorname{Spec}\left(A_{x}^{\text {int }} \otimes_{\mathcal{O}} k\right)$, choose a Frobenius lift $\sigma$ on $A_{x}$ and choose a lift $u_{x}$ of $t_{x}$ in $A_{x}^{\text {int }}$. Let $A_{x}^{\prime}$ be the localization of $A_{x}$ at $u_{x}$; then the restriction of $\mathcal{E}$ to $U \backslash\{x\}$ corresponds to a $(\sigma, \nabla)$-module $M_{x}$ over $A_{x}^{\prime}$. After shrinking $U_{x}$ if needed, we may assume that $M_{x}$ is free over $A_{x}^{\prime}$.

Let $\rho_{x}$ be the embedding of $A_{x}$ into $\mathcal{R}^{+ \text {,int }}$ sending $u_{x}$ to the series parameter. By Proposition 3.1, there exists a nearly finite separable extension $L_{x}$ of the $t_{x^{-}}$ adic completion of $K(X)$ such that $M_{x} \otimes_{A_{x}^{\prime}} \mathcal{R}_{L_{x}}^{\text {int }}$ is unipotent. By Krasner's lemma, after replacing $k$ with $k^{1 / p^{n}}$ for some nonnegative integer $n$, we can choose a finite separable extension $L$ of $K(X)$ whose completion at any point above $x$ contains $L_{x}$ for each $x \in Z$. After enlarging $k$ again, we may assume that the places of $L$ above $x$ are all $k$-rational. Let $f: \overline{X_{1}} \rightarrow \bar{X}$ be the cover corresponding to the extension $L / K(X)$, and put $X_{1}=f^{-1}(X)$.

For each geometric point $y$ of $Z_{1}=\overline{X_{1}} \backslash X_{1}$, choose a function $t_{y} \in K\left(X_{1}\right)$ with a simple zero at $y$ and no multiple zeroes; then $t_{y}$ gives rise to a map $g_{y}: \overline{X_{1}} \rightarrow \mathbb{P}^{1}$. (If $k$ is finite, it may be necessary to enlarge it again to find such $t_{y}$.) Put $x=f(y)$, and choose an open affine neighborhood $V_{y}$ of $y$ in $\overline{X_{1}}$ such that $V_{y} \cap Z_{1}=\{y\}, \operatorname{div}\left(t_{y}\right) \cap V_{y}=\{y\}, f\left(V_{y}\right) \subseteq U_{x}$, and $V_{y}$ does not meet the branch locus of $g_{y}$. Then there is a dagger algebra $B_{y}$ with $V_{y} \cong \operatorname{Spec}\left(B_{y}^{\text {int }} \otimes_{\mathcal{O}} k\right)$ which is a localization of a finite extension of $A_{x}$.

Choose a lift $u_{y}$ of $t_{y}$ in $B_{y}^{\text {int }}$. Since $V_{y}$ is unramified over its image under $g_{y}$, $B_{y}$ is finite and unramified over some localization $C_{y}$ of its subring $K\left\langle u_{y}\right\rangle^{\dagger}$ (the $p$-adic closure of $K\left[u_{y}\right]$ within $B_{y}$ ). Now $K\left\langle u_{y}\right\rangle^{\dagger}$ admits a $p$-power Frobenius 
lift $\sigma_{0}^{\prime}$ sending $u_{y}$ to $u_{y}^{\sigma}$; this lift extends to the localization $C_{y}$, then to the unramified extension $B_{y}$. Put $\sigma^{\prime}=\left(\sigma_{0}^{\prime}\right)^{a}$.

Let $B_{y}^{\prime}$ be the localization of $B_{y}$ at $u_{x} \in A_{x} \subseteq B_{y}$, which is the same as the localization at $u_{y}$ because $\operatorname{div}\left(t_{x}\right) \cap V_{y}=\operatorname{div}\left(t_{y}\right) \cap V_{y}=\{y\}$, and put $V_{y}^{\prime}=\operatorname{Spec}\left(\left(B_{y}^{\prime}\right)^{\mathrm{int}} \otimes_{\mathcal{O}} k\right)=V_{y} \backslash\{y\}$; then the restriction of $f^{*} \mathcal{E}$ to $V_{y}^{\prime}$ corresponds to the $\left(\sigma^{\prime}, \nabla\right)$-module $M_{x} \otimes_{A_{x}^{\prime}} B_{y}^{\prime}$. (That is, its connection is the one induced from $M_{x}$, but its Frobenius structure is defined with respect to $\sigma^{\prime}$ instead of $\sigma$.) By construction, this $\left(\sigma^{\prime}, \nabla\right)$-module is unipotent. Moreover, $u_{y}^{\sigma^{\prime}} / u_{y}^{q}=1$, so Theorem 5.1 implies that $M_{x} \otimes_{A_{x}^{\prime}} B_{y}^{\prime}$ is isomorphic to a $\log -(\sigma, \nabla)$-module over some localization $B_{y}^{\prime \prime}$ of $B_{y}$ in which $u_{y}$ is not invertible. If $V_{y}^{\prime \prime}=\operatorname{Spec}\left(\left(B_{y}^{\prime \prime}\right)^{\text {int }} \otimes_{\mathcal{O}}\right.$ $k$ ), then $V_{y}^{\prime \prime}$ is an open affine neighborhood of $y$ in $\overline{X_{1}}$ on which $f^{*} \mathcal{E}$ extends to a $\log -F$-isocrystal.

In short, we have an open affine neighborhood of each $y \in Z_{1}$ in $\overline{X_{1}}$, on which $f^{*} \mathcal{E}$ extends to a $\log -F$-isocrystal relative to $\{y\}$. Each neighborhood contains no other points of $y$, so the pairwise intersections all lie in $X_{1}$. Thus we automatically have glueing isomorphisms on the $\log -F$-isocrystals satisfying the cocycle conditions (since $f^{*} \mathcal{E}$ is defined on $X_{1}$ ), yielding a log- $F$-isocrystal on $\left(\overline{X_{1}}, Z_{1}\right)$, as desired.

\section{Finite versus étale}

As noted earlier, Crew [Cr, Section 10] conjectured that an overconvergent $F$-isocrystal on a curve should extend to a log- $F$-isocrystal after a base extension which is not just finite and generically étale, but actually étale. It is unclear whether this should hold in general; the best we can do at the moment is prove it in the unit-root case, as done below. Note that this proof does not use the full strength of the quasi-unipotence theorem, but only the unit-root case; this case is due to Tsuzuki [T1]. Also note that a unit-root log- $F$-isocrystal is automatically an $F$-isocrystal, so there is no "log" in the statement of the theorem.

The proof of the following lemma is straightforward.

Lemma 6.1. Let $B$ be a matrix over $k[[t]]$, for $k$ a perfect field of characteristic $p>0$, and let $\tau$ denote the $q$-th power map. Then any solution $D$ of either of the matrix equations

$$
D^{-1} B D^{\tau}=I \quad \text { or } \quad D^{\tau}-D=B
$$

over the integral closure of $k[[t]]$ in $k((t))^{\text {alg }}$ is defined over an unramified extension of $k[[t]]$.

Theorem 6.2. Let $X$ be a smooth, geometrically connected curve over a perfect field $k$ of characteristic $p>0$, and let $\mathcal{E}$ be an overconvergent unit-root $F^{a}$ isocrystal on $X / K$. Then there exists a finite étale morphism $f: X_{1} \rightarrow X, a$ smooth compactification $j: X_{1} \hookrightarrow \overline{X_{1}}$ of $X_{1}$, and a unit-root $F^{a}$-isocrystal $\mathcal{F}$ on $\overline{X_{1}}$ such that $j^{*} \mathcal{F} \cong f^{*} \mathcal{E}$. 
Proof. If $X$ is projective, there is nothing to prove, so we assume $X$ is affine. Let $A$ be a dagger algebra with $X \cong \operatorname{Spec}\left(A^{\text {int }} \otimes_{\mathcal{O}} k\right)$, and choose a Frobenius lift $\sigma$ on $A$. Then $\mathcal{E}$ corresponds to a $(\sigma, \nabla)$-module $M$ over $A$. Choose (not necessarily free) generators $\mathbf{v}_{1}, \ldots, \mathbf{v}_{m}$ of $M$, and let $N \subset M$ be the $A^{\text {int }}$-span of $F^{i} \mathbf{v}_{j}$ over $i=0,1, \ldots$ and $j=1, \ldots, m$. Then $N$ is locally free over $A^{\text {int }}$.

Let $L$ be the $p$-adic completion of the valuation subring of Frac $A$; note that $N$ is free over $L$ and $F$ acts on any basis of $N$ over $L$ via an invertible matrix. Let $\pi$ be a uniformizer of $\mathcal{O}$, and pick an integer $d$ such that $v_{p}\left(\pi^{d}\right)>1 /(p-1)$.

Given any basis $\mathbf{e}_{1}, \ldots, \mathbf{e}_{n}$ of $N$ to start with, define the matrix $\Phi$ by $F \mathbf{e}_{j}=$ $\sum_{i j} \Phi_{i j} \mathbf{e}_{i}$. We then solve the matrix equation $C_{i}^{-1} \Phi C_{i}^{\sigma} \equiv I\left(\bmod \pi^{i}\right)$ for $i=$ $1, \ldots, d$ to obtain a matrix $C_{d}$ over some finite unramified extension $L^{\prime}$ of $L$. Then $N \otimes_{A^{\text {int }}} L^{\prime}$ admits a basis $\mathbf{w}_{1}, \ldots, \mathbf{w}_{n}$ for which $F \mathbf{w}_{i} \equiv \mathbf{w}_{i}\left(\bmod \pi^{d}\right)$.

If we insist that $L^{\prime}$ be minimal for the existence of the basis of the desired form, then it is unique; in particular, it does not depend on the choice of the starting basis. Let $X_{1}$ be a curve for which $K\left(X_{1}\right) \cong L^{\prime} / \pi L^{\prime}$ and let $f: X_{1} \rightarrow X$ be the induced map. If we choose the initial basis $\mathbf{e}_{1}, \ldots, \mathbf{e}_{n}$ over a localization $A_{1}^{\text {int }}$ of $A^{\text {int }}$ over which $N$ becomes free, we discover that $f$ is étale over any point in $\operatorname{Spec}\left(A_{1}^{\text {int }} \otimes_{\mathcal{O}} k\right)$ by Lemma 6.1. (Namely, $C_{0}$ satisfies an equation modulo $\pi$ of the first type in the lemma, while $C_{i-1}^{-1} C_{i}=I+\pi^{i-1} D$ for some matrix $D$ satisfying an equation modulo $\pi$ of the second type.) Since $N$ is locally free over $A^{\text {int }}$, we can arrange for $\operatorname{Spec}\left(A_{1}^{\text {int }} \otimes_{\mathcal{O}} k\right)$ to contain any closed point of $X$. Thus $f$ is finite étale. Since $v_{p}\left(\pi^{d}\right)>1 /(p-1)$, we may apply [T1, Theorem 5.1.1] (at least for $a=1$; see [Ke2, Proposition 6.11] for a reduction to this case) to see that $f^{*} \mathcal{E}$ admits a basis of elements in the kernel of $\nabla$, on which $F$ acts by a matrix over $\mathcal{O}$. This allows us to extend $f^{*} \mathcal{E}$ to a compactification $\overline{X_{1}}$ of $X_{1}$, as desired.

\section{Acknowledgments}

Thanks to Pierre Berthelot, Johan de Jong and Fabien Trihan for helpful discussions. The author was partially supported by a National Science Foundation Postdoctoral Fellowship.

\section{References}

[A] Y. André, Filtrations de type Hasse-Arf et monodromie p-adique, Invent. Math. 148 (2002), 285-317.

[Be] P. Berthelot, Géométrie rigide et cohomologie des variétés algebriques de caractéristique $p$, Introductions aux cohomologies $p$-adiques (Luminy, 1984). Mém. Soc. Math. France 23 (1986), 7-32.

[Bo] N. Bourbaki, Algebre Commutative, Chap. IX-X, Masson (Paris), 1983.

[CI] R. Coleman, A. Iovita, Hidden structures on semistable curves, preprint: math. berkeley.edu/ coleman

[Cr] R. Crew, Finiteness theorems for the cohomology of an overconvergent isocrystal on a curve, Ann. Sci. École Norm. Sup. (4) 31 (1998), 717-763.

[dJ] A. J. de Jong, Barsotti-Tate groups and crystals, Proceedings of the International Congress of Mathematicians, Vol. II (Berlin, 1998). Doc. Math. 1998, Extra Vol. II, 259-265. 
[Ke1] K.S. Kedlaya, Descent theorems for overconvergent F-crystals, PhD thesis, Massachusetts Institute of Technology, 2000.

[Ke2] — A p-adic local monodromy theorem, to appear in Ann. Math.; arXiv: math.AG/0110124

[Ke3] - Finiteness of rigid cohomology with coefficients, preprint; arXiv: math.AG/0208027

[M] Z. Mebkhout, Analogue p-adique du théorème de Turrittin et le théorème de la monodromie p-adique, Invent. Math. 148 (2002), 319-351.

[S] A. Shiho, Crystalline fundamental groups. II. Log convergent cohomology and rigid cohomology, J. Math. Sci. Univ. Tokyo 9 (2002), 1-163.

[T1] N. Tsuzuki, Finite local monodromy of overconvergent unit-root F-isocrystals on a curve, Amer. J. Math. 120 (1998), 1165-1190.

[T2] Slope filtration of quasi-unipotent overconvergent F-isocrystals, Ann. Inst. Fourier (Grenoble) 48 (1998), 379-412.

[vdP] M. van der Put, The cohomology of Monsky and Washnitzer, Introductions aux cohomologies p-adiques (Luminy, 1984), Mém. Soc. Math. France 23 (1986), 33-60.

Department of Mathematics, University of California, Berkeley, Berkeley, CA 94720, U.S.A.

E-mail address: kedlaya@math.berkeley.edu 